PROCEEDINGS OF THE

AMERICAN MATHEMATICAL SOCIETY

Volume 124, Number 1, January 1996

\title{
ON THE CLASSIFICATION OF PYRAMIDAL CENTRAL CONFIGURATIONS
}

\author{
NELLY FAYÇAL
}

(Communicated by Hal L. Smith)

\begin{abstract}
We present some results associated with the existence of central configurations (c.c.'s) in the classical gravitational $N$-body problem of Newton. We call a central configuration of five bodies, four of which are coplanar, a pyramidal central configuration (p.c.c). It can be shown that there are only three types of p.c.c.'s, admitting one or more planes of symmetry, viz. (i) the case where the planar bodies lie at the vertices of a regular trapezoid, (ii) the case where the bodies lie at the vertices of a kite-shaped quadrilateral, and (iii) the case where the bodies lie at the vertices of a rectangle. In this paper we classify all p.c.c.'s with a rectangular base and, in fact, prove that there is only one such c.c., namely, the square-based pyramid with equal masses at the corners of the square. The classification of all p.c.c.'s satisfying either (i) or (ii) will be discussed in subsequent papers.
\end{abstract}

\section{INTRODUCTION}

Let $\bar{q}_{1}, \ldots, \bar{q}_{N} \in R^{3}$ denote the position vectors of $N$ bodies with masses $m_{1}, \ldots$, $m_{N}$ respectively subject to the influence of Newton's law of gravitation. The motion of the system is then governed by the relations

$$
m_{i} \ddot{\bar{q}}_{i}=\frac{\partial U}{\partial \bar{q}_{i}}, 1 \leq i \leq N
$$

where

$$
U=\sum_{1 \leq i<j \leq N} \frac{m_{j} m_{i}}{\left|\bar{q}_{j}-\bar{q}_{i}\right|}
$$

is the potential energy of the system.

Consider the space

$$
X=\left\{\bar{q}=\left(\bar{q}_{1}, \ldots, \bar{q}_{N}\right) \in R^{3 N} ; \sum_{i=1}^{N} m_{i} \bar{q}_{i}=0\right\},
$$

i.e., suppose that the center of mass is fixed at the origin of the space. Let $\triangle=$ $\bigcup_{i \leq j} \triangle_{i j}$ be the set of diagonals where

$$
\triangle_{i j}=\left\{\bar{q}=\left(\bar{q}_{1}, \ldots, \bar{q}_{N}\right) \in R^{3 N} ; \bar{q}_{i}=\bar{q}_{j}\right\}, 1 \leq i, j \leq N .
$$

The set $X \backslash \triangle$ is called the configuration space.

Received by the editors July 25, 1994.

1991 Mathematics Subject Classification. Primary 70F15, 70F10.

This research was partially supported by an NSERC, PGS3 Scholarship.

(C)1996 American Mathematical Society 
Definition 1.1. A point $\bar{q}=\left(\bar{q}_{1}, \ldots, \bar{q}_{N}\right) \in X \backslash \triangle$ is a central configuration (c.c.) if there exists a constant $\lambda$ such that

$$
\sum_{\substack{j=1 \\ j \neq i}}^{N} \frac{m_{j} m_{i}}{\left|\bar{q}_{j}-\bar{q}_{i}\right|^{3}}\left(\bar{q}_{j}-\bar{q}_{i}\right)=-\lambda m_{i} \bar{q}_{i}, 1 \leq i \leq N .
$$

The value of the constant $\lambda$ in (3) is uniquely determined by the formula, given by Wintner [9],

$$
\lambda=-\frac{U}{J}
$$

where $U$ is given by (2) and

$$
J=\sum_{i=1}^{N} m_{i}\left|\bar{q}_{i}\right|^{2}
$$

Moreover, if $A$ is an orthogonal matrix, then clearly $A \bar{q}=\left(A \bar{q}_{1}, \ldots, A \bar{q}_{N}\right)$ is also a central configuration with the same $\lambda$. If $\kappa \neq 0$, then $\kappa \bar{q}=\left(\kappa \bar{q}_{1}, \ldots, \kappa \bar{q}_{N}\right)$ is also a central configuration with $\lambda$ replaced by $\lambda / \kappa^{3}$. Thus any configuration similar to a central configuration, either by rescaling or rotation, is also a central configuration. When counting central configurations one counts only similarity classes.

Definition 1.2. A central configuration of five bodies, four of which are coplanar, the fifth being off the plane, is called a pyramidal central configuration (p.c.c.). Equivalently, we will say that the c.c. has the shape of a pyramid.

The problem of the existence of c.c.'s dates back to the 18th century, when, in 1767, Euler [2] discovered collinear c.c.'s when $N=3$. According to Wintner [9], it was Lagrange [3], and not Laplace, who in 1772 first proved that, for any three arbitrary masses, there exists exactly one c.c.: the equilateral triangle. The corresponding question for $N=4$ was considered by Lehmann-Filhés [4], who showed that the regular tetrahedron is a c.c. for any choice of the masses. This work was completed by Pizzetti in 1904, who, in his pioneering paper [7], showed that, in fact, the regular tetrahedron is the only non-flat c.c. corresponding to four arbitrary masses. Aside from these fundamental results little else is known in terms of a classification of c.c.'s for given $N \geq 4$. Indeed, even in the flat case when $N=4$, we do not have a complete classification of the shapes of c.c.'s, although there are some results (e.g., lower bounds) on the number of such c.c.'s.

It is the scope of this paper to contribute some results on the classification of c.c.'s in the 5-body problem. However, some restrictions on the initial positions of the given bodies are necessary at this point in our research, as is to be expected. More specifically, we will limit our efforts to the problem of determining the shape of all the c.c.'s of five bodies, four of which lie on a common plane, and admitting one or more planes of symmetry: Even with these restrictions, the calculations are very technical.

It can be shown that there are only three types of p.c.c.'s, admitting one or more planes of symmetry, viz. (i) the case where the planar bodies lie at the vertices of a regular trapezoid, (ii) the case where the bodies lie at the vertices of a kite-shaped quadrilateral, and (iii) the case where the bodies lie at the vertices of a rectangle.

In this paper we classify all p.c.c.'s with a rectangular base and, in fact, prove that there is only one such c.c., namely, the square-based pyramid with equal masses 
at the corners of the square. The classification of all p.c.c.'s satisfying either (i) or (ii) will be discussed in subsequent papers.

For further results on c.c.'s see [8] where Schmidt characterizes some c.c.'s of five bodies using, after Dziobek, the mutual distances between the masses as coordinates. Since Dziobek's coordinates are singular when four bodies lie in a plane, we see that our results cannot be derived using the same method.

\section{The mutual Distances}

Consider five bodies with masses $m_{1}, \ldots, m_{5}>0$ in a Newtonian field. Suppose that $m_{1}, \ldots, m_{5}$ are at the vertices $P_{1}, \ldots, P_{5}$ of a rectangular-based pyramid such that $m_{5}$ is at the top vertex and $m_{1}, \ldots, m_{4}$ are at the vertices of the base. Without loss of generality, we can assume that they are in counterclockwise order (Figure 1). Let $\bar{q}_{1}, \ldots, \bar{q}_{5}$ denote the position vectors of $m_{1}, \ldots, m_{5}$ in a coordinate system $G \bar{x} \bar{y} \bar{z}$ whose origin is at the center of mass of the five bodies. By Definition 1.1, $\bar{q}=\left(\bar{q}_{1}, \ldots, \bar{q}_{5}\right)$ forms a c.c. then there exists a scalar $\lambda$ such that

$$
\sum_{\substack{j=1 \\ j \neq i}}^{5} \frac{m_{j} m_{i}}{\left|\bar{q}_{j}-\bar{q}_{i}\right|^{3}}\left(\bar{q}_{j}-\bar{q}_{i}\right)=-\lambda m_{i} \bar{q}_{i}, \quad 1 \leq i \leq 5 .
$$

Writing $\bar{q}_{i}=\left(\bar{x}_{i}, \bar{y}_{i}, \bar{z}_{i}\right) \in R^{3}$, in terms of its coordinates, and $D_{j i}=\left|\bar{q}_{j}-\bar{q}_{i}\right|$ for $1 \leq i, j \leq 5$, we obtain

$$
\sum_{\substack{j=1 \\ j \neq i}}^{5} \frac{m_{j}}{D_{j i}^{3}}\left(\bar{x}_{j}-\bar{x}_{i}\right)=-\lambda \bar{x}_{i}, 1 \leq i \leq 5 .
$$

This expression was apparently first derived by Pizzetti [7] in his pioneering work on homographic solutions of non-flat problems.

The masses $m_{1}, \ldots, m_{4}$ lie on a common plane. We may assume then, without loss of generality, that this plane is parallel to $G \bar{y} \bar{z}$. Hence, $\bar{x}_{1}=\bar{x}_{2}=\bar{x}_{3}=\bar{x}_{4}$. Inserting these relations into (6), we readily obtain

$$
D_{15}=D_{25}=D_{35}=D_{45}
$$

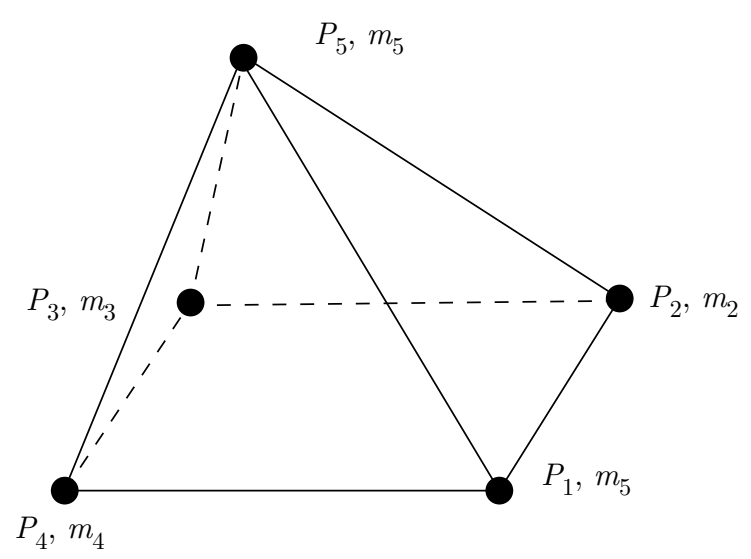

Figure 1 
Thus, we have proved

Lemma 2.1. Let $\bar{q}=\left(\bar{q}_{1}, \ldots, \bar{q}_{5}\right)$ be a p.c.c. with a rectangular base such that $m_{5}$ is at the top vertex and $m_{1}, \ldots, m_{4}$ are at the vertices of the base. Then $m_{5}$ is equidistant from $m_{1}, m_{2}, m_{3}$ and $m_{4}$.

This surprising fact is a key observation that will reduce considerably the complexity of the computations in our problem.

Remark. The masses $m_{1}, \ldots, m_{4}$ are concyclic.

In fact, they lie on the intersection of a plane with a sphere, for they are coplanar by assumption, and they belong to a sphere centered at $m_{5}$ by Lemma 2.1 .

Since the notion of a c.c. is independent of the orientation of the system of coordinates, we can now suppose, without loss of generality, that the plane containing the masses $m_{1}, m_{2}, m_{5}$ is parallel to $G \bar{y} \bar{z}$, i.e., $\bar{x}_{1}=\bar{x}_{2}=\bar{x}_{5}$. Hence, (4) gives for $i=1,2,5$

where

$$
\begin{aligned}
\frac{1}{8} m_{3}\left(\bar{x}_{3}-\bar{x}_{1}\right)+b m_{4}\left(\bar{x}_{4}-\bar{x}_{1}\right) & =-\lambda \bar{x}_{1}, \\
b m_{3}\left(\bar{x}_{3}-\bar{x}_{1}\right)+\frac{1}{8} m_{4}\left(\bar{x}_{4}-\bar{x}_{1}\right) & =-\lambda \bar{x}_{1}, \\
g m_{3}\left(\bar{x}_{3}-\bar{x}_{1}\right)+g m_{4}\left(\bar{x}_{4}-\bar{x}_{1}\right) & =-\lambda \bar{x}_{1},
\end{aligned}
$$

$$
a=\frac{1}{D_{12}^{3}}=\frac{1}{D_{34}^{3}}, b=\frac{1}{D_{14}^{3}}=\frac{1}{D_{23}^{3}},
$$

and

$$
g=\frac{1}{D_{5 i}^{3}}, 1 \leq i \leq 4 \text {. }
$$

Note. $g$ is determined uniquely by Lemma 2.1.

The system (7)-(9) can be transformed into a homogeneous system, linear in $m_{3}\left(\bar{x}_{3}-\bar{x}_{1}\right)$ and $m_{3}\left(\bar{x}_{4}-\bar{x}_{1}\right)$, by taking the difference $((7)-(8))$ and $((8)-(9))$. A necessary condition for the existence of solutions is

$$
\left(\frac{1}{8}-b\right)\left(\frac{1}{8}+b-2 g\right)=0
$$

If we repeat the same process for the masses $\left(m_{1}, m_{4}, m_{5}\right)$ we get

$$
\left(\frac{1}{8}-a\right)\left(\frac{1}{8}+a-2 g\right)=0
$$

But $D_{12}, D_{14} \neq 2$, hence comparing (10) and (11) we get that

$$
a=b
$$

or

$$
D_{12}=D_{14}
$$

Then the rectangular base is necessarily a square.

Since a c.c. is invariant under rescaling, we can assume, without loss of generality, that the square base of the pyramid is inscribed in a circle of diameter $\sqrt{2}$. Hence,

$$
D_{12}=D_{14}=1 \text {. }
$$

Now that we have $a=1$, equation (10) gives

$$
D_{51}=\frac{2}{\sqrt[3]{4+\sqrt{2}}}
$$


Therefore,

Lemma 2.2. Every p.c.c. with a rectangular base is necessarily similar (up to isometry) to a pyramid with a square base, where the mutual distances are given by

$$
\begin{aligned}
& D_{12}=D_{23}=D_{34}=D_{41}=1, \\
& D_{13}=D_{24}=\sqrt{2},
\end{aligned}
$$

and

$$
D_{5 i}=\frac{2}{\sqrt[3]{4+\sqrt{2}}}, 1 \leq i \leq 4
$$

\section{The MASSES}

Similar to our approach in the previous section on the mutual distances, we will consider a p.c.c. with a rectangular base as desribed at the beginning of Section 2 and find necessary conditions on the masses.

Without loss of generality, we may assume that $G \bar{x} \bar{y}$ is parallel to the plane $P$ containing $m_{1}, \ldots, m_{4}$.

Formula (4), given by Wintner to determine the value of the scalar $\lambda$ in Definition 1.1, is not computationally practical. For the most part it leads very complicated expressions. However, our choice of the coordinate system $G \bar{x} \bar{y} \bar{z}$ will allow us to overcome this problem and find a simplified form for $\lambda$ which will reveal to be of great importance in establishing necessary conditions on the masses $m_{1}, \ldots, m_{5}$. In fact, we have

Lemma 3.1. If $\bar{q}=\left(\bar{q}_{1}, \ldots, \bar{q}_{5}\right)$ is a p.c.c. with a rectangular base, then

$$
\lambda=m g
$$

where $m=m_{1}+\ldots+m_{5}$ is the total mass and $g=1 / D_{5 i}^{3}, 1 \leq i \leq 4$.

Moreover, for this choice of $\lambda$ and $G \bar{x} \bar{y} \bar{z}$ (G $\bar{x} \bar{y}$ parallel to the plane containing $\left.m_{1}, \ldots, m_{4}\right)$, the equations

$$
\sum_{\substack{j=1 \\ j \neq i}}^{5} \frac{m_{j} m_{i}}{\left|\bar{q}_{j}-\bar{q}_{i}\right|^{3}}\left(\bar{q}_{j}-\bar{q}_{i}\right) \cdot \vec{k}=-\lambda m_{i} \bar{q}_{i} \cdot \vec{k}, 1 \leq i \leq 5,
$$

where $\vec{k}$ is the unit vector in the $\bar{z}$-direction, are satisfied for $1 \leq i \leq 5$.

Proof. Denote by $O x y z$, the coordinate system obtained from $G \bar{x} \bar{y} \bar{z}$ by parallel translation to a new origin $O \in P$ (Figure 2 on the next page). Let $q_{1}, \ldots, q_{5}$ be the position vectors of $m_{1}, \ldots, m_{5}$ in Oxyz.

Obviously,

$$
\overrightarrow{O G}=\frac{1}{m} \sum_{j=1}^{5} m_{j} q_{j}
$$

$\bar{q}=\left(\bar{q}_{1}, \ldots, \bar{q}_{5}\right)$ is a c.c. Then by definition (5) is satisfied. Taking the scalar multiple of (5) with $\vec{k}$ for $1 \leq i \leq 4$, and using $\bar{q}_{i}=q_{i}-\overrightarrow{O G}$, we get

$$
\sum_{\substack{j=1 \\ j \neq i}}^{5} \frac{m_{j} m_{i}}{\left|\bar{q}_{j}-\bar{q}_{i}\right|^{3}}\left(\bar{q}_{j}-\bar{q}_{i}\right) \cdot \vec{k}=-\lambda m_{i}\left(q_{i}-\overrightarrow{O G}\right) \cdot \vec{k} .
$$




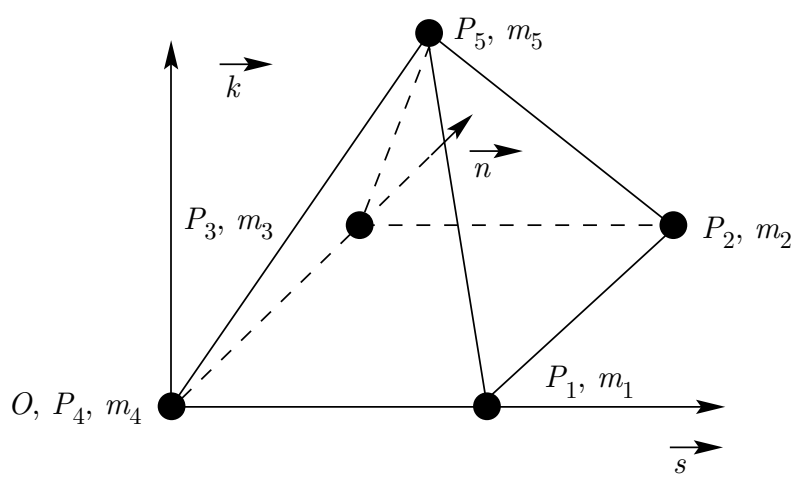

Figure 2

But $\vec{k}$ is perpendicular to the plane $P$ containing the vectors $q_{i}, 1 \leq i \leq 4$; then using (13) equation (14) gives

$$
\frac{m_{5}}{D_{5 i}^{3}} q_{5} \cdot \vec{k}=\frac{\lambda}{m} m_{5} q_{5} \cdot \vec{k}
$$

Hence,

$$
\lambda=\frac{m}{D_{5 i}^{3}}, 1 \leq i \leq 4 .
$$

On the other hand, for $i=5$, the left-hand side of (5) gives

$$
\begin{aligned}
\sum_{\substack{j=1 \\
j \neq 5}}^{5} \frac{m_{j} m_{5}}{\left|\bar{q}_{j}-\bar{q}_{5}\right|^{3}}\left(\bar{q}_{j}-\bar{q}_{5}\right) \cdot \vec{k} & =\sum_{j=1}^{4} \frac{m_{j} m_{5}}{\left|q_{j}-q_{5}\right|^{3}}\left(q_{j}-q_{5}\right) \cdot \vec{k} \\
& =-\frac{1}{D_{j 5}^{3}}\left(\sum_{j=1}^{4} m_{j}\right) m_{5} q_{5} \cdot \vec{k} .
\end{aligned}
$$

Now, the right-hand side of (5) gives

$$
\begin{aligned}
-\lambda m_{5} \bar{q}_{5} \cdot \vec{k} & =-\lambda m_{5}\left(q_{5}-\overrightarrow{O G}\right) \cdot \vec{k}=-\lambda \frac{m_{5}\left(m-m_{5}\right)}{m} q_{5} \cdot \vec{k} \\
& =-\frac{m_{5}\left(m-m_{5}\right)}{D_{5 i}^{3}} q_{5} \cdot \vec{k} .
\end{aligned}
$$

Since (15) and (16) are clearly equal, (5) is satisfied for $1 \leq i \leq 5$.

Now, we are ready to prove

Lemma 3.2. Given a p.c.c. with a rectangular base for the masses $m_{1}, \ldots, m_{5}$ such that $m_{5}$ is at the top vertex of the pyramid and $m_{1}, \ldots, m_{4}$ are at the vertices of the base, then

(i) $m_{5}$ is arbitrary, and

(ii) $m_{1}=m_{2}=m_{3}=m_{4}$. 
Proof. Let $\bar{q}=\left(\bar{q}_{1}, \ldots, \bar{q}_{5}\right)$ be a p.c.c. for the masses $m_{1}, \ldots, m_{5}$. There exists $\lambda$ such that (5) is satisfied. Following the notation introduced in Section 2, (5) is equivalent to

$$
\sum_{\substack{j=1 \\ j \neq i}}^{5} \frac{m_{j}}{D_{j i}^{3}}\left(q_{j}-q_{i}\right)=-\lambda \bar{q}_{i},
$$

for $1 \leq i \leq 5$.

(i) Using $\bar{q}_{i}=q_{i}-\overrightarrow{O G}, \lambda=\frac{m}{D_{51}^{3}}$ and (13), (17) gives

$$
\sum_{\substack{j=1 \\ j \neq i}}^{5} \frac{m_{j}}{D_{j i}^{3}}\left(q_{j}-q_{i}\right)=\sum_{\substack{j=1 \\ j \neq i}}^{5} \frac{m_{j}}{D_{51}^{3}}\left(q_{j}-q_{i}\right)
$$

for $1 \leq i \leq 5$. Hence,

$$
\sum_{\substack{j=1 \\ j \neq i}}^{4} m_{j}\left(\frac{1}{D_{j i}^{3}}-\frac{1}{D_{51}^{3}}\right)\left(q_{j}-q_{i}\right)=0
$$

because $D_{j i}=D_{51}$ for $j=5$.

Therefore, (5) is equivalent to (18), which is independent of $m_{5}$. Hence, $m_{5}$ can be taken as arbitrary. We note that (18) is identically satisfied for $i=5$.

(ii) Let $\vec{n}$ be a unit vector perpendicular to the line segment $P_{1} P_{4}$ on the base of the pyramid.

Taking the scalar multiple of (17) with $\vec{n}$, we get for $i=1$

$$
\frac{m_{2} h}{D_{21}^{3}}+\frac{m_{3} h}{D_{31}^{3}}+\frac{m_{5}}{D_{51}^{3}}\left(q_{5}-q_{1}\right) \cdot \vec{n}=-\lambda \bar{q}_{1} \cdot \vec{n},
$$

and for $i=4$

$$
\frac{m_{2} h}{D_{24}^{3}}+\frac{m_{3} h}{D_{31}^{3}}+\frac{m_{5}}{D_{54}^{3}}\left(q_{5}-q_{4}\right) \cdot \vec{n}=-\lambda \bar{q}_{4} \cdot \vec{n},
$$

where $h=D_{12}=D_{34}$.

Since $h \neq 0, D_{21}=D_{34}$ and $D_{31}=D_{24}$, equating (19) and (20) gives

$$
\left(m_{2}-m_{3}\right)\left(\frac{1}{D_{21}^{3}}-\frac{1}{D_{31}^{3}}\right)=0 .
$$

Hence, $m_{2}=m_{3}$ because $D_{21} \neq D_{31}$, otherwise $m_{1}$ and $m_{4}$ collide.

Similarly, we prove that $m_{1}=m_{4}, m_{1}=m_{2}$ and $m_{3}=m_{4}$. Therefore,

$$
m_{1}=m_{2}=m_{3}=m_{4} .
$$

\section{The mutual Distances: another approach}

The results in Section 3 were developed independently of Section 2. This was done on purpose in order to illustrate a second approach to finding the mutual distances between the masses.

In fact, assume we have proved Lemma 2.1, Lemma 3.1 and Lemma 3.2 and that we are trying to compute the mutual distances of a p.c.c. with a rectangular base. 
Let $\vec{n}$ and $\vec{s}$ be the unit vectors respectively perpendicular and parallel to the line segment $P_{1} P_{4}$. Taking the scalar product of (3), where $N=5$, with $\vec{n}$ and $\vec{s}$ respectively, we get for $i=1$

$$
m_{1} \sum_{j=2}^{5} \frac{\left(\bar{q}_{j}-\bar{q}_{1}\right) \cdot \vec{n}}{D_{j 1}^{3}}=-\lambda \bar{q}_{1} \cdot \vec{n}
$$

and

$$
m_{1} \sum_{j=2}^{5} \frac{\left(\bar{q}_{j}-\bar{q}_{1}\right) \cdot \vec{s}}{D_{j 1}^{3}}=-\lambda \bar{q}_{1} \cdot \vec{s},
$$

since $m_{1}=m_{2}=m_{3}=m_{4}$ by Lemma 3.2. If we let

$$
h=D_{12}, H=D_{14}, \text { and } D=D_{13},
$$

then (21) and (22) imply

$$
\frac{1}{h^{3}}+\frac{1}{D^{3}}=\frac{\lambda}{2 m_{1}}
$$

and

$$
\frac{1}{D^{3}}+\frac{1}{H^{3}}=\frac{\lambda}{2 m_{1}}
$$

since $h, H$, and $m_{1} \neq 0$. Comparing (23) and (24), we get

$$
h=H \text {. }
$$

Therefore, $D_{12}=D_{14}$, i.e., the rectangular base is necessarily a square. The rest of the proof is identical to what was presented in Section 2.

To recapitulate, we have proved so far that if we are given a p.c.c. with a rectangular base, then

(i) The pyramid is necessarily similar to one with a square base and with mutual distances described in Lemma 2.2 .

(ii) The masses are such that

$$
\begin{aligned}
& m_{5} \text { is arbitrary, and } \\
& m_{1}=m_{2}=m_{3}=m_{4} .
\end{aligned}
$$

Conversely, suppose we are given the five masses $m_{1}, \ldots, m_{5}$ at the vertices of a square-based pyramid with mutual distances given by Lemma 2.2 and such that the masses $m_{1}, \ldots, m_{4}$ at the vertices of the base are equal and the mass $m_{5}$ at the top vertex is arbitrary as in (ii).

Consider a coordinate system $G \bar{x} \bar{y} \bar{z}$ with origin at the center of mass of $m_{1}, \ldots$, $m_{5}$ such that $G \bar{x}$ is parallel to $P_{4} P_{1}$ and $G \bar{y}$ is parallel to $P_{4} P_{3}$. Since $m_{1}=m_{2}=$ $m_{3}=m_{4}$, their center of mass is at the center of the square base. Hence, $G$ is on the line segment joining $P_{5}$ to the center of the square.

Let $\bar{q}_{i}$ be the position vector of $m_{i}, 1 \leq i \leq 5$, in the coordinate system $G \bar{x} \bar{y} \bar{z}$. By Definition 1.2, we need to find $\lambda$ such that (5) is satisfied. Now, let $O x y z$ be the parallel translate of $G \bar{x} \bar{y} \bar{z}$ with origin $O$ at $P_{4}$.

By Lemma 3.1, we can choose

$$
\lambda=\frac{m}{D_{5 i}^{3}}
$$


where $m=m_{1}+\ldots+m_{5}=4 m_{1}+m_{5}$ so that (12) is automatically satisfied. Let $q_{i}$ be the position vector of $m_{i}, 1 \leq i \leq 5$, in Oxyz. Equation (12) is equivalent to

$$
\sum_{\substack{j=1 \\ j \neq i}}^{5} \frac{m_{j}}{D_{j i}^{3}}\left(q_{j}-q_{i}\right) \cdot \vec{k}=-\lambda \bar{q}_{i} \cdot \vec{k}
$$

for $1 \leq i \leq 5$. Hence, we need only to check that equation (27) is satisfied for the $\bar{x}, x$ and $\bar{y}, y$ components, $1 \leq i \leq 5$.

For this, in the rest of the proof, $\bar{q}_{i}, q_{i}$ will denote the projections of the position vectors of $m_{i}, 1 \leq i \leq 5$, on the $G \bar{x} \bar{y}, O x y$ planes respectively:

$$
q_{1}=\left(\begin{array}{l}
1 \\
0
\end{array}\right), q_{2}=\left(\begin{array}{l}
1 \\
1
\end{array}\right), q_{3}=\left(\begin{array}{l}
0 \\
1
\end{array}\right), q_{4}=\left(\begin{array}{l}
0 \\
0
\end{array}\right)
$$

and

$$
q_{5}=\left(\begin{array}{c}
1 / 2 \\
1 / 2
\end{array}\right)
$$

In the proof of Lemma 3.2 we saw that the definition of a c.c. given by equation (3) is equivalent to equation (18), in the case we are studying. Using the symbolic computation software "Maple V" [1], we wrote a procedure $\mathrm{c}(\mathrm{m}, \mathrm{d}, \mathrm{q})$ which yields a matrix of $i$ th row equal to

$$
\sum_{\substack{j=1 \\ j \neq i}}^{4} m_{j}\left(\frac{1}{D_{j i}^{3}}-\frac{1}{D_{51}^{3}}\right)\left(q_{j}-q_{i}\right)
$$

where $\mathrm{m}=\left(m_{1}, \ldots, m_{5}\right)$ denotes the vector of masses, $\mathrm{d}=\left(D_{i j}\right)_{1 \leq i, j \leq 5}$ denotes the matrix of mutual distances, and $\mathrm{q}=\left(q_{1}, \ldots, q_{5}\right)^{\text {transpose }}$ denotes the matrix of position row vectors.

Since all the entries of the matrix c(m, d, q) are 0, we conclude that equation (18) is satisfied and hence, the pyramid described in (i) and (ii) of Section 5 forms a c.c.

Hence, we have proved

Theorem 4.1. The only p.c.c. with a rectangular base is (up to isometry) the square-based pyramid with mutual distances

$$
\begin{aligned}
& D_{12}=D_{23}=D_{34}=D_{41}=1, \\
& D_{13}=D_{24}=\sqrt{2},
\end{aligned}
$$

and

$$
D_{5 i}=\frac{2}{\sqrt[3]{4+\sqrt{2}}}, 1 \leq i \leq 4
$$

where the masses are taken such that the mass $m_{5}$ at the top vertex is arbitrary and the masses on the base are equal, i.e., $m_{1}=m_{2}=m_{3}=m_{4}$.

Finally, in 1990, Moeckel [6] considered four equal masses on the vertices of a square and an arbitrary fifth mass on the top vertex of a regular pyramid based on this square. He proved that there exists a height for which the pyramid forms a central configuration for this choice of masses. Our work exhibits this pyramid (i.e., it is possible to calculate the value of the height) and proves its uniqueness. Moreover, it proves that the choice of equal masses on the vertices of the square base is not only sufficient but also necessary. 


\section{ACKNOWLEDGMENT}

The author wishes to express her sincere thanks to Professor Angelo B. Mingarelli for getting her started on the problem, and for his constant help and encouragement.

\section{REFERENCES}

1. B.W. Char, K.O. Geddes, G.H. Gonnet, B.L. Leong, M.B. Monagan, and S.M. Watt, First leaves: A tutorial introduction to Maple V, Springer-Verlag, New York, 1992.

2. L. Euler, De mot rectilineo trium corporum se mutuo attahentium, Novi Comm. Acad. Sci. Imp. Petrop. 11 (1767), 144-151.

3. J.L. Lagrange, Oeuvres, vol. 6, Gauthier-Villars, Paris, 1873, 272-292.

4. Lehmann-Filhés, Ueber zwei Fälle des Vielkörperproblems, Astr. Nachr. 127 (1891), 137-144.

5. K.R. Meyer and G.R. Hall, Introduction to Hamiltonian dynamical systems and the $N$ body problem, Appl. Math. Sci., vol. 90, Springer-Verlag, New York and Berlin, 1992. MR 93b:70002

6. R. Moeckel, On central configurations, Math. Z. 205 (1990), 499-517. MR 92b:70012

7. P. Pizzetti, Casi particolari del problema dei tre corpi, Rendiconti 13 (1904), 17-26.

8. D. S. Schmidt, Central configurations in $R^{2}$ and $R^{3}$, Contemp. Math., vol. 81, Amer. Math. Soc., Providence, RI, 1988, pp. 59-76 MR 90d:70028.

9. A. Wintner, The analytical foundations of celestial mechanics, Princeton Univ. Press, Princeton, NJ, 1941. MR 3:215b

Carleton University, Department of Mathematics and Statistics, Ottawa, Ontario, CANADA K1S 5B6

E-mail address: nfaycal@math.carleton.ca 